\title{
THE JOURNAL OF PHYSICAL CHEMISTRY
}

Subscriber access provided by UNIV OF SOUTHAMPTON

\section{C: Plasmonics; Optical, Magnetic, and Hybrid Materials}

\author{
All-Fiber Plasmonic Platform Based on \\ Hybrid Composite Metal/Glass Microwires
}

Afroditi Petropoulou, Grigoris Antonopoulos, Paul Bastock, George Kakarantzas, Christopher Craig, Daniel W. Hewak, Michalis N. Zervas, and Christos Riziotis

J. Phys. Chem. C, Just Accepted Manuscript • DOI: 10.1021/acs.jpcc.8b08844 • Publication Date (Web): 19 Oct 2018

Downloaded from http://pubs.acs.org on October 23, 2018

\section{Just Accepted}

"Just Accepted" manuscripts have been peer-reviewed and accepted for publication. They are posted online prior to technical editing, formatting for publication and author proofing. The American Chemical Society provides "Just Accepted" as a service to the research community to expedite the dissemination of scientific material as soon as possible after acceptance. "Just Accepted" manuscripts appear in full in PDF format accompanied by an HTML abstract. "Just Accepted" manuscripts have been fully peer reviewed, but should not be considered the official version of record. They are citable by the Digital Object Identifier (DOI@). "Just Accepted" is an optional service offered to authors. Therefore, the "Just Accepted" Web site may not include all articles that will be published in the journal. After a manuscript is technically edited and formatted, it will be removed from the "Just Accepted" Web site and published as an ASAP article. Note that technical editing may introduce minor changes to the manuscript text and/or graphics which could affect content, and all legal disclaimers and ethical guidelines that apply to the journal pertain. ACS cannot be held responsible for errors or consequences arising from the use of information contained in these "Just Accepted" manuscripts. 


\title{
All-Fiber Plasmonic Platform Based on Hybrid Composite
}

\section{Metal/Glass Microwires}

\author{
Afroditi Petropoulou ${ }^{1,2}$, Grigoris Antonopoulos ${ }^{1}$, Paul Bastock ${ }^{3}$, George Kakarantzas ${ }^{1}$, Christopher \\ Craig $^{3}$, Daniel W Hewak ${ }^{3}$, Michalis N Zervas ${ }^{3}$, and Christos Riziotis*1 \\ ${ }^{1}$ Theoretical and Physical Chemistry Institute, National Hellenic Research Foundation, Athens 11635, \\ Greece \\ ${ }^{2}$ Department of Informatics and Telecommunications, University of Peloponnese, Tripolis 22100, Greece \\ ${ }^{3}$ Optoelectronics Research Centre, University of Southampton, Southampton SO17 1BJ, United Kingdom \\ * Corresponding author: Christos Riziotis, E-mail: Riziotis@eie.gr Tel: +302107273887
}




\begin{abstract}
Metal tips are emerging plasmonic structures that can offer high field intensity at the tip apex and high confinement in the nanoscale. The fabrication though of smooth metal tips with well-defined geometrical characteristics, crucial for optimizing the performance of the plasmonic structure, is not trivial. Furthermore pure metal tips are exposed to the environment and fragile, thus, complicating their use in real applications. The proposed platform based on hybrid composite glass metal microwires can offer the required robustness for device development. An optimized fabrication process of high quality all-fiber plasmonic tips by tapering such hybrid metal core/dielectric cladding microfibers is proposed and demonstrated experimentally. The presence of the dielectric cladding offers continuous re-excitation of the plasmon modes due to repeated total internal reflection at the glass/air interface which can dramatically reduce the high losses induced by the metal core. This enables direct light coupling from the distal end of fiber instead of side excitation of the tip, allowing thus their integration in optical fiber and planar circuits. Plasmonic tips were successfully demonstrated in a highly controllable manner and their performance was related to simulation results predicting high field enhancement factors up to $10^{5}$.
\end{abstract}




\section{INTRODUCTION}

Surface plasmon polaritons (SPP's) which are electromagnetic waves travelling along metal/dielectric interfaces can focus the light beyond the diffraction limit enhancing its intensity by several orders of magnitude. ${ }^{1,2}$ The high confinement of the electromagnetic energy in the nanoscale is crucial for many applications such as sensing, ${ }^{3-7}$ surface enhanced Raman spectroscopy (SERS), ${ }^{8,9}$ data storage $^{10}$ and microscopy ${ }^{11,12}$ since it can offer high sensitivity and improve the detection limits. Different structures that support plasmonic modes have been investigated in various platforms such as metal/dielectric interfaces of planar $^{13,14}$ and cylindrical geometry. ${ }^{15,16}$ The special category of optical fiber-based plasmonic devices ${ }^{17}$ provides a platform of unique advantages as it offers connection with single mode fibers, ${ }^{18}$ ease of light coupling, and excitation of plasmon resonances. ${ }^{19-21}$

Metal tips ${ }^{22-25}$ are very promising nanofocusing structures since they enable the confinement of light at the tip apex providing strong field localization. In this work microwires composed of noble metal cores with glass cladding ${ }^{26}$ are proposed and employed for the development of plasmonic metal tips. The glass cladding not only mechanically supports and shields the metal core, but also due to the total internal reflections at the glass/air interface the plasmon modes travelling along the metal/dielectric interface are continuously re-excited. ${ }^{27}$ This is similar to range extension of surface plasmons by dielectric layers, ${ }^{28}$ an approach which has been proven quite efficient in the design and implementation of integrated devices with incorporated plasmonic structures. ${ }^{29,30}$ The re-excitation of the plasmon modes up to the tip apex is crucial for limiting the losses induced by the metal. This is an important improvement compared to previously published works where the metal tip is formed outside the glass cladding. ${ }^{23}$ This all-fiber plasmonic device offers ease of light coupling directly from the distal end of the fiber instead of side excitation of the tip. It also allows the direct integration into standard optical fiber via fiber splicing techniques.

In the present work the tips are fabricated by tapering hybrid metal core/glass cladding microfibers. The heating and stretching method, ${ }^{31,32}$ a simple and cost-effective technique followed previously ${ }^{26}$ was further 
optimized by the study of time-transient effects associated to core break-up, obtaining thus proper conditions of flame's temperature and velocity for the development of smooth and adiabatic tips. The obtained tapering conditions controlled the break-up process of the metal core and the formation of microsphere features due to various intrinsic and external instabilities. To our knowledge, the process of fully controllable down tapering of hybrid metal/glass fibers by means of the heating and stretching method is here experimentally demonstrated for the first time.

Simulations of metal tips with glass claddings were also performed through Finite Element Method (FEM) using the commercially available COMSOL Multiphysics modelling software for the calculation of the intensity enhancement at the tip apex. Additionally the near field distributions at the end-faces of tapered and untapered fibers were calculated theoretically and were compared with the experimental results.

\section{RESULTS AND DISCUSSION}

\section{A. Fabrication of Hybrid Metal Core/Glass Cladding Microfibers}

The hybrid gold core/borosilicate cladding microfibers were fabricated in a variety of sizes -as the fiber drawing process is not yet standardized- while in this study were considered microfibers with typical core and cladding diameters of $4 \mu \mathrm{m}$ and $30 \mu \mathrm{m}$, respectively. ${ }^{33}$ For the glass cladding, Schott Duran borosilicate tubing ( $81 \mathrm{wt} \% \mathrm{SiO}_{2}, 13 \mathrm{wt} \% \mathrm{~B}_{2} \mathrm{O}_{2}, 4 \mathrm{wt} \% \mathrm{Na}_{2} \mathrm{O}+\mathrm{K}_{2} \mathrm{O}, 2 \mathrm{wt} \% \mathrm{Al}_{2} \mathrm{O}_{3}$ ) was chosen due to the compatibility of its softening and working points which are $825{ }^{\circ} \mathrm{C}$ and $1260{ }^{\circ} \mathrm{C}$, respectively, with the melting point of the metals used. Tubes of $6.00 \mathrm{~mm}$ and $2.2 \mathrm{~mm}$ outer and inner diameter, respectively, were filled with four strands of metal wire each $0.5 \mathrm{~mm}$ in diameter. Fibers were drawn at a furnace temperature of $1310{ }^{\circ} \mathrm{C}$ in an argon purged tube furnace with hot zone of $50 \mathrm{~mm}$ length. ${ }^{33}$ The metals used were silver, copper, gold and aluminum having melting points ranging from $660{ }^{\circ} \mathrm{C}$ for aluminum to 1085 ${ }^{\circ} \mathrm{C}$ for copper (Figure 1). In this work only gold core microfibers were studied due to various current fabrication restrictions affecting microwires' core uniformity. 

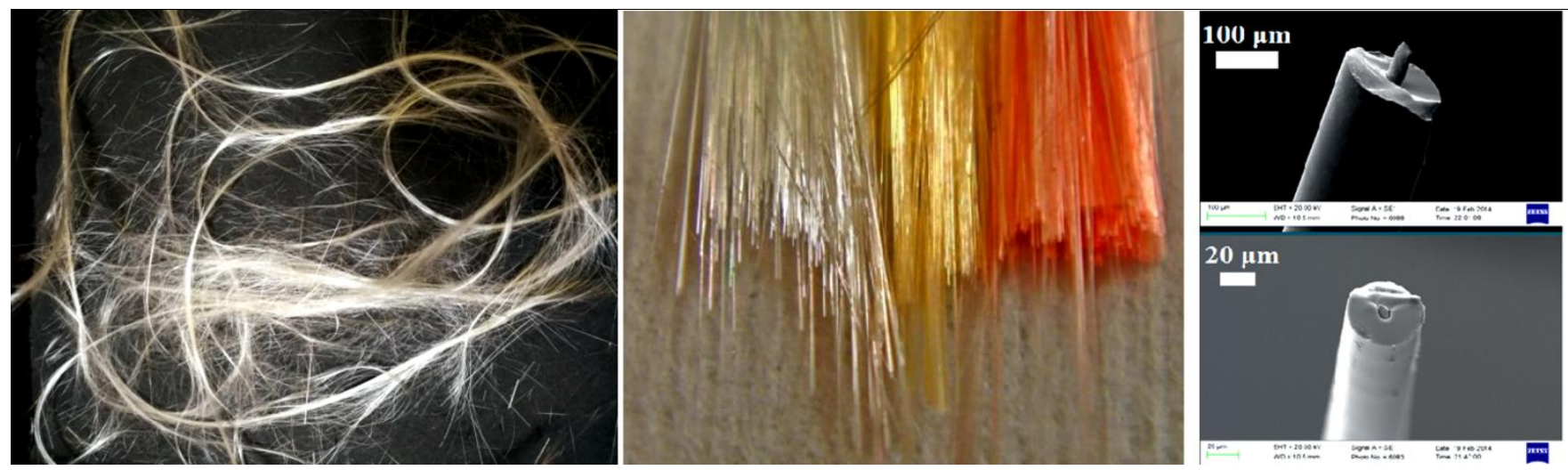

Figure 1. From left to right: Pristine form of continuous long length gold core microwires. Bundles of silver, gold and copper-core borosilicate microfibers. SEM images of metal-core borosilicate microfibers.

The main parameters that were controlled during the drawing process of the fibers in order to obtain microfibers with smooth and continuous metal cores are the temperature, feed rate, pull rate and volume of the used metal. Although after the optimization of the aforementioned parameters and the fabrication of high quality microfibers, certain random discontinuities appear in the metal core of the fibers currently limiting the length available for processing. These discontinuities do not alter the mode profile at the output of the fiber due to the repeated re-excitation of the fundamental mode but they affect the tapering process as for discontinuous cores the glass collapses during the tapering. Due to the current restriction on available continuous core fibers, only microfibers segments of limited length and with continuous cores were processed and tapered in the present study. Specifically fiber pieces of length $\sim 12 \mathrm{~cm}$ which contained microfiber segments with continuous cores of length $\sim 2-3 \mathrm{~cm}$ at least, were carefully selected. The $12 \mathrm{~cm}$ length facilitated initial waveguiding characterization experiments while the continuous microfiber segments of $\sim 2-3 \mathrm{~cm}$ length were used for the thermal tapering process. Work towards the optimization of the drawing process in order to establish core continuity over long microwire lengths is currently in progress. 


\section{B. Study of Microwires' Modal Characteristics}

Theoretical studies were performed for the calculation of the fundamental $\mathrm{TM}_{01}$ mode profile of hybrid microfibers as well as for the field enhancement at the tip apex using Finite Element Methods FEM by the commercial software package COMSOL Multiphysics Modelling software. A typical microfiber with a 4 $\mu \mathrm{m}$ gold core diameter and a $30 \mu \mathrm{m}$ borosilicate cladding was simulated. The excitation wavelength is 532 $\mathrm{nm}$ and it was chosen for direct comparison with the experimental measurements. The calculated electric field of the $\mathrm{TM}_{01}$ mode is concentrated at the metal surface and decays exponentially far from it, forming two intense peaks corresponding to the metal/glass interface (Figure 2).

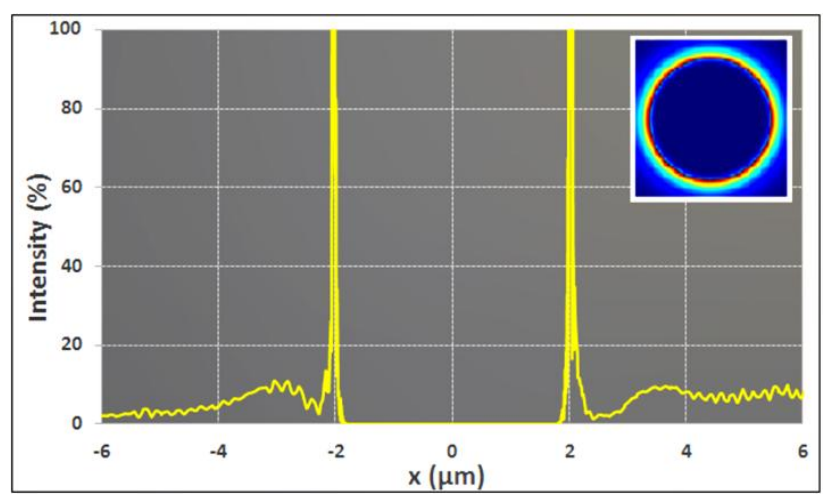

Figure 2. TM $M_{01}$ mode profile of a microfiber with $4 \mu \mathrm{m}$ gold core diameter and a 30 um borosilicate cladding. Inset picture: $2 D$ representation of the $T M_{01}$ mode profile.

The effective refractive index of the $\mathrm{TM}_{01}$ mode for gold core/borosilicate cladding microfibers was calculated for different core radii (Figure 3) using the dispersion equation ${ }^{34}$ :

$$
\begin{aligned}
& \varepsilon_{1} \alpha_{2} I_{1}\left(\alpha_{1} R_{1}\right)\left[\alpha_{2} \varepsilon_{3} K_{1}\left(\alpha_{3} R_{2}\right) M_{00}+\alpha_{3} \varepsilon_{2} K_{0}\left(\alpha_{3} R_{2}\right) M_{10}\right]=-\varepsilon_{2} \alpha_{1} I_{0}\left(\alpha_{1} R_{1}\right)\left[\alpha_{2} \varepsilon_{3} K_{1}\left(\alpha_{3} R_{2}\right) M_{01}+\right. \\
& \left.\alpha_{3} \varepsilon_{2} K_{0}\left(\alpha_{3} R_{2}\right) M_{11}\right]
\end{aligned}
$$

with $M_{a b}=I_{a}\left(a_{2} R_{2}\right) K_{b}\left(a_{2} R_{1}\right)-(-1)^{a+b} I_{b}\left(a_{2} R_{1}\right) K_{a}\left(a_{2} R_{2}\right)$ 
Here $\varepsilon_{1}, \varepsilon_{2}$ and $\varepsilon_{3}$ are the relative permittivities of gold core, borosilicate cladding and air, respectively, $\alpha_{\mathrm{j}}$ $=\left(\beta^{2}-\varepsilon_{\mathrm{j}} k^{2}\right)^{1 / 2}$ for $\mathrm{j}=1,2,3, \beta$ is the propagation constant, $I$ and $K$ are the modified Bessel functions of the first and second kind and $\mathrm{R}_{1}$ and $\mathrm{R}_{2}$ are the radii of the core and cladding, respectively.

The borosilicate cladding is large enough $\left(\mathrm{r}_{\text {cladding }}>10 \mu \mathrm{m}\right)$ and its exact radius does not influence the $\mathrm{n}_{\text {eff }}$ since the field for $\mathrm{TM}_{01}$ mode is concentrated at the metal/glass interface as presented in Figure 2. As the core radius decreases both real and imaginary part of $n_{\text {eff }}$ increase leading to significant losses for small core radius.
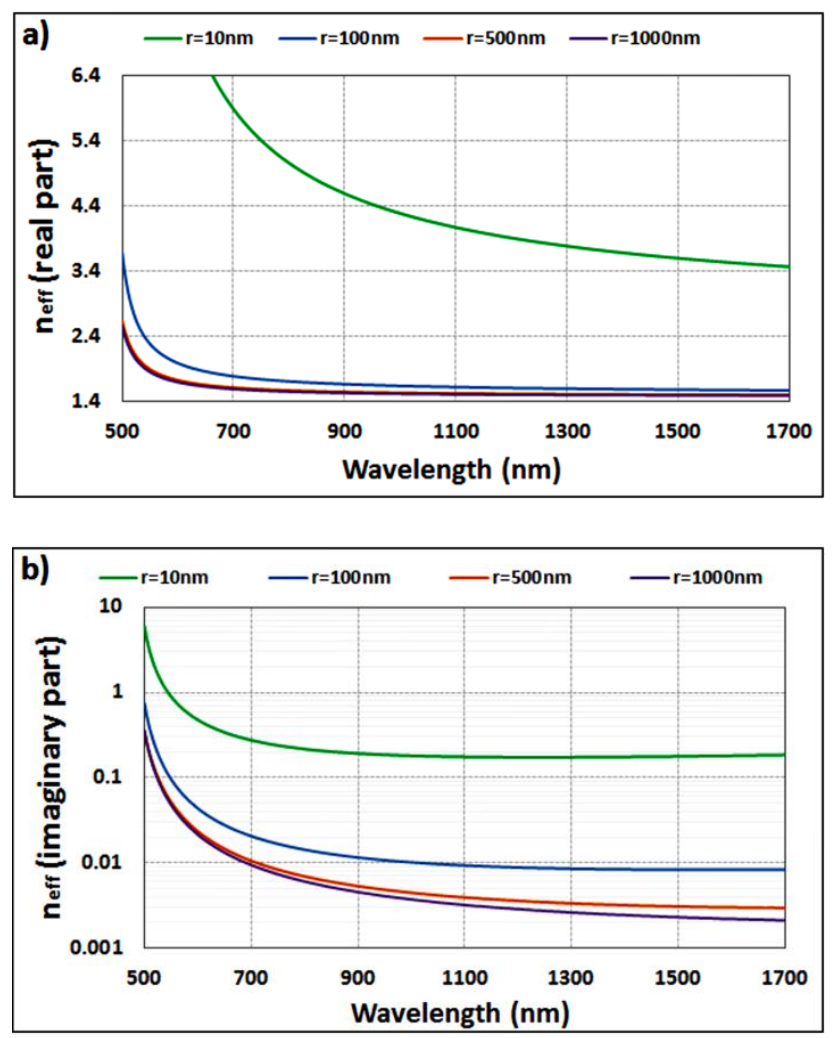

Figure 3. Real (a) and imaginary (b) part of the effective refractive index $\left(n_{\text {eff }}\right)$ of $T M_{01}$ mode as a function of the wavelength for different radii of the gold core.

\section{3D Simulations of Metal Tips}

A gold core/borosilicate cladding tapered microfiber was simulated in order to calculate the intensity enhancement at the tip apex for $532 \mathrm{~nm}$ excitation wavelength. Since the fabricated tapered microfibers 
have semi-angles between $3^{\circ}-4^{\circ}$ for both core and cladding, as it will be discussed later, the simulated tip has semi-angle $\mathrm{a}=3.5^{\circ}$. For these semi-angles the adiabaticity condition $^{35}$ :

$$
\left|\frac{d\left(\beta_{r}\right)^{-1}}{d z}\right| \ll 1
$$

where $\beta_{\mathrm{r}}$ is the real part of the propagation constant, is satisfied and thus the reflections of the SPP's that can lead to significant reduction of the intensity enhancement at the tip apex are limited. For computer memory efficiency purposes only the last $2.4 \mu \mathrm{m}$ of the tip was simulated. Furthermore in order to reduce calculation time only half of the structure was simulated with the separatrix being set to be a perfect magnetic conductor in order to launch the fundamental $\mathrm{TM}_{01}$ mode. In the simulated limited segment the input core radius is set to $150 \mathrm{~nm}$ and at the tip apex is $3 \mathrm{~nm}$ in order to avoid computational singularities. The core is surrounded by a large enough borosilicate glass layer and the whole structure is surrounded by a sufficient large air box with Scattering Boundary Conditions applied at its exterior boundaries to avoid reflections. Boundary mode analysis was used for launching the $\mathrm{TM}_{01}$ mode and the input power was $1 \mathrm{~W}$.
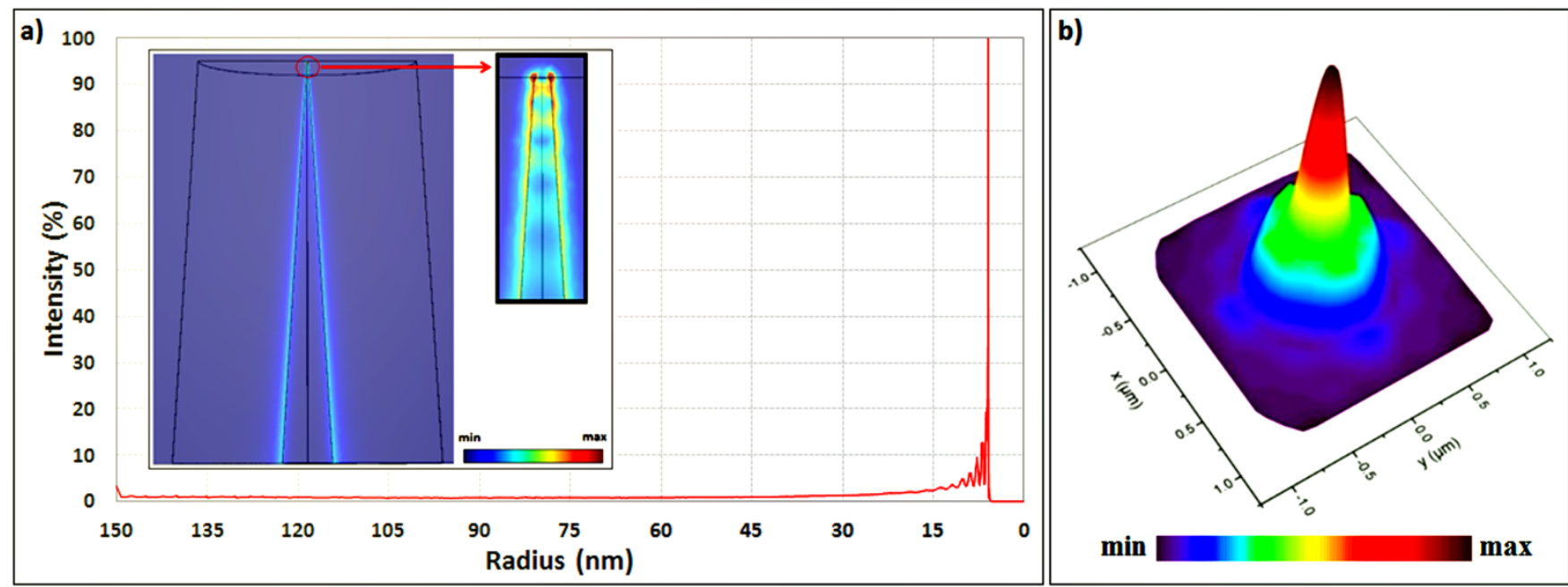

Figure 4. a) Field intensity along the metal/glass interface. Inset picture: Zoom-in of the normalized intensity at the tip apex. b) Near field distribution at the tip apex. 
Figure 4 (a) and (b) show the field intensity along the metal/glass interface and the near at the tip apex respectively. The inset picture of Figure 4 (a) shows the field intensity at the tapered core surface. High field enhancement and localization is achieved at the tip apex due to the excited SPP's since plasmon modes do not suffer from cut-offs contrary to photonic modes propagating in optical fibers. The intensity enhancement, calculated as:

$$
E_{f}=\frac{I_{\text {apex }}}{I_{0}}=\frac{\left|E_{\text {apex }}\right|^{2}}{\left|E_{0}\right|^{2}}
$$

Here $\mathrm{I}_{\text {apex }}, \mathrm{I}_{0}, \mathrm{E}_{\text {apex }}, \mathrm{E}_{0}$ are the intensity and electric field at the tip apex and input, respectively, is $\sim 10^{3}$. As discussed above, due to the high losses of the $\mathrm{TM}_{01}$ mode for small core radii, i.e., $\mathrm{r}<200 \mathrm{~nm}$, the propagation length, defined as the distance the SPP travels before its intensity is reduced by a factor of e, is less than $4 \mu \mathrm{m}$ for $\lambda=532 \mathrm{~nm}$. Hence the re-excitation of the $\mathrm{TM}_{01}$ mode from the light reflected at the glass/air interface is crucial in order to obtain a high $E_{f}$. Higher $E_{f}$ up to $\sim 10^{5}$ can also be achieved for longer excitation wavelengths that exhibit lower losses as seen in Figure 5 where the maximum electric field at the tip apex $\left(E_{\max }\right)$ as a function of the wavelength is presented, which is comparable or even higher than previously published reports. ${ }^{36,37}$ The dependency of the maximum electric field at the tip apex can be physically associated to the $\mathrm{TM}_{01}$ mode dispersion relation as this is calculated form Eq. 1 and shown graphically in Fig. 3. As can be seen in Fig 3b the propagation losses of $\mathrm{TM}_{01}$-as indicated by $\operatorname{Im}\left(\mathrm{n}_{\mathrm{eff}}\right)$ dependency- are higher for lower excitation wavelengths which is in agreement with the dependency of $E_{\max }$ as illustrated at Fig. 5. The oscillatory dependence -periodic peaks- appeared in the spectrum and in the spatial field distribution along the fiber originates from the cavity modes formed due to the reflection of the propagating SPPs at the tip apex. ${ }^{38}$ Another factor that determines the $E_{f}$ is the core radius at the tip apex. As the core radius decreases both group and phase velocities of the propagating SPP's decrease leading to the observed high field concentration. Hence the highest possible $E_{f}$ corresponds to a core radius close to zero. 


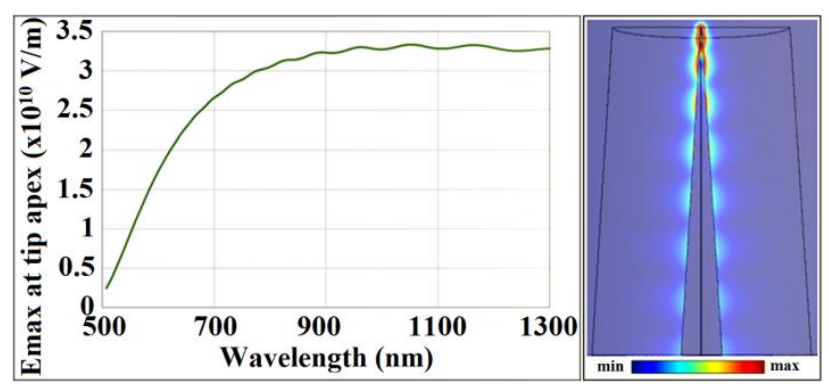

Figure 5. Left: Maximum normalized electric field at the tip apex as a function of the wavelength. Right: Normalized electric field for $1200 \mathrm{~nm}$ excitation wavelength along a tapered microfiber.

\section{Fabrication of Metal Core/ Glass Cladding Tips}

For the tapering process the heating and stretching processing method was chosen as the more suitable and flexible for tapering the gold core microfibers. The microfiber is placed on two linear stages that stretch it while a third stage moves the flame along the fiber axis (Figure 6). The tapering system is computer controlled via LabView software to accurately move the stages in order to obtain the desired geometrical characteristics of the tapered microfiber. The appropriate temperature is achieved by adjusting the distance between the flame and the microfiber as well as its thermal profile by controlling the ratio of the butane - oxygen mixture using two electronic mass flow controllers.

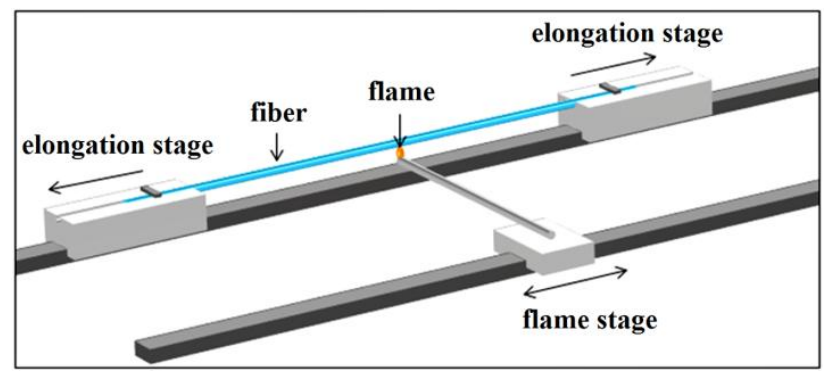

Figure 6. Schematic representation of the experimental setup for heating and stretching based tapering. The system is composed by a set of two linear translation stages for fiber stretching and one independent translation stage for flame positioning control. 
However, it should be stressed that various conditions in the tapering process of such delicate fibers have imposed difficulties in smooth tapers fabrication. During the experimental tapering process it was noticed that breakup of the inner metal core or also the formation of isolated microspheres (Figure 7) could easily occur in un-optimized conditions. This could be attributed to instabilities induced by the temperature profile of the flame or by spatial irregularities of the core's surface. Such instabilities in fluidic systems have been investigated in various material systems ${ }^{39-41}$ and in fiber platforms. ${ }^{42,43}$ The key parameter describing the instability phenomena is the break up time $\tau_{\mathrm{B}}$, of the continuous medium which can be quantitatively estimated using the Tomotika model instability rate (INR) ${ }^{44}$ :

$$
\mathrm{INR}=\frac{\mathrm{s}}{2 \mathrm{ru}_{\text {glass }}}\left(1-\mathrm{x}_{\mathrm{m}}{ }^{2}\right) \Phi\left(\mathrm{x}_{\mathrm{m}}\right)
$$

Here, $s$ is the surface tension, $r$ the core radius, $\mu_{\text {glass }}$ is the glass viscosity, $x=\frac{2 \pi r}{\lambda}$ with $\lambda$ being the wavelength of the induced variation and $x_{m}$ is the value of parameter $x$ that maximizes $\left(1-x^{2}\right) \Phi(x)$ with $\Phi(x)$ being a function of $x$.

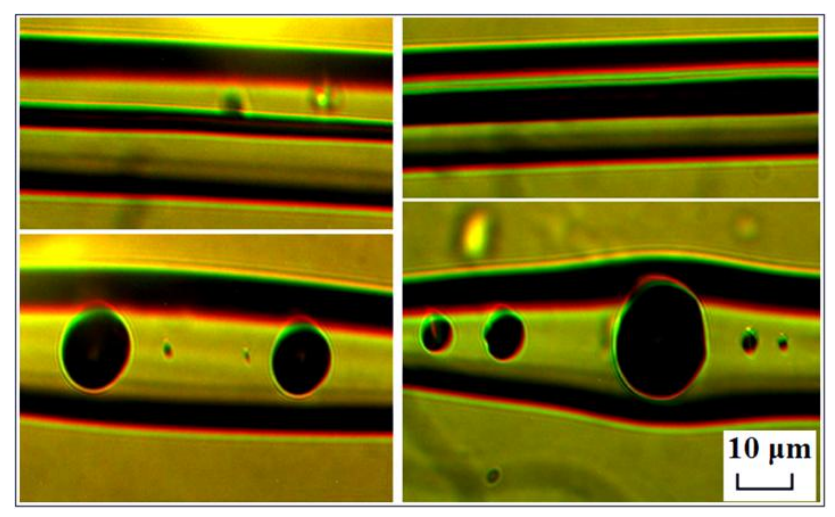

Figure 7. Microscope images of formed spheres from microfibers with initial core radius of $2 \mu \mathrm{m}$ (left) and $3 \mu m$ (right). 
The fact that in the case studied here the glass viscosity is very large compared to the metal viscosity, makes the factor $\frac{s}{2 r \mu_{\text {glass }}}$ the dominant term of the instability rate resulting to a calculated $\tau_{\mathrm{B}}$ less than 1 second. As the temperature increases, $\tau_{\mathrm{B}}$ decreases due to the corresponding decrease in $\mu_{\text {glass. }}$ In order to accommodate those time-scale restrictions an approach for fast moving of flame (flame velocity of $6 \mathrm{~mm} / \mathrm{s}$ ) with the lowest possible temperature of flame (close to $1065{ }^{\circ} \mathrm{C}$ which is the melting point of gold) was followed for the tapering of the microfibers in order to avoid the collapse of the core. We will refer to this approach from now on as a "fast and cold" method.

The aforementioned experimental observations of in-fibre structures formation were also connected to theoretical investigations by transient microfluidics FEM simulations (performed by COMSOL Microfluidics module) for the determination and validation of the appropriate conditions of the proposed tapering process. Since the temperature of the flame is the most crucial parameter, the tapering process for two different temperatures, $1065^{\circ} \mathrm{C}$ and $1200{ }^{\circ} \mathrm{C}$, was simulated (Figure 8). The simulated segment was 60 $\mu \mathrm{m}$ long and the core radius was $2 \mu \mathrm{m}$ while a sinusoidal perturbation was applied to the surface tension in order to introduce the required instability to the system. The simulation time interval was set to $0.3 \mathrm{~s}$ since the model does not converge for longer time scales due to its computational complexity in this specific materials platform. However due to the high flame velocity, $0.3 \mathrm{~s}$ provides sufficient time for a substantial length of fiber to withdraw from the heat zone of the flame. As expected in the case of "cold" tapering, at $1065{ }^{\circ} \mathrm{C}$, the microfiber is tapered down without breaking, while at $1200{ }^{\circ} \mathrm{C}$ the fiber core breaks into spheres as observed in Figure 8. 


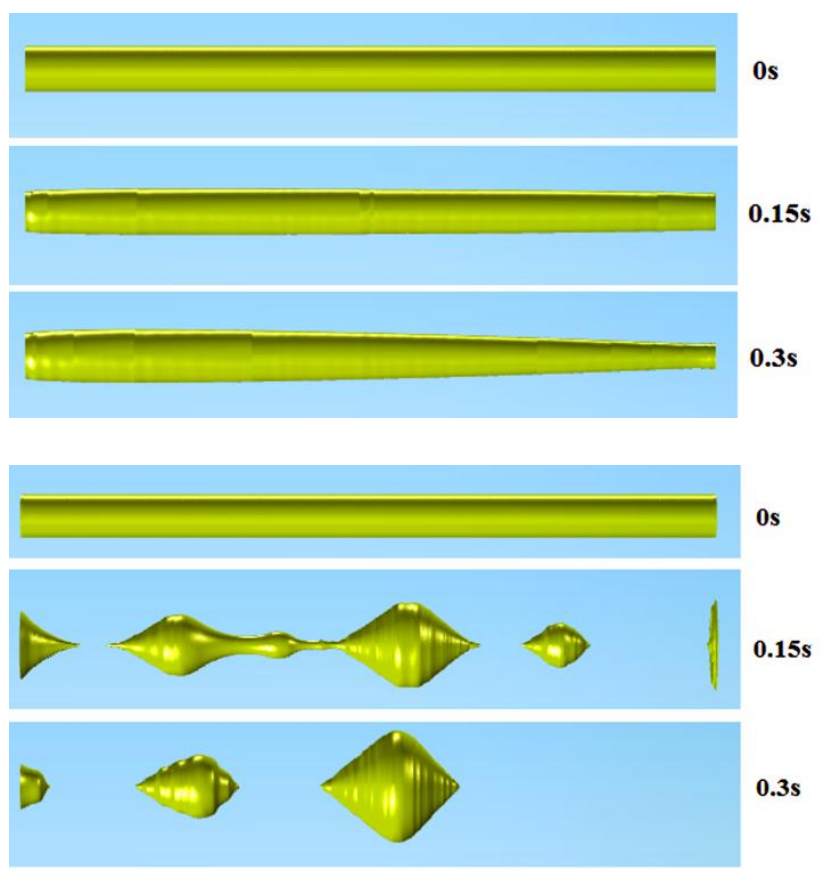

Figure 8. Simulation of the tapering process of a $2 \mu \mathrm{m}$ core radius microfiber for $1065{ }^{\circ} \mathrm{C}$ (up) and 1200 ${ }^{\circ} \mathrm{C}$ (down) showing the core's shape evolution. At $1065{ }^{\circ} \mathrm{C}$ the core remains solid while at $1200{ }^{\circ} \mathrm{C}$ the core breaks into isolated spheres. Although the formed spheres exhibit a conical-like shape attributed to a transient phase of the simulation process, the metal core breakage is obvious.

Although, the scope of this work is the fabrication of uniformly tapered metal tips, the study of microspheres' formation, potentially as embedded optical micro-resonators can be also very interesting in terms of photonics applications. The study of the mechanism and the dynamics of instabilities driven microfluidics phenomena in such hybrid glass/noble metal systems are currently under consideration and will be reported elsewhere.

\section{E. Characterization of Metal Core/ Glass Cladding Tips}

During the experimental procedure the key tapering parameters such flame temperature and speed were carefully optimized and selected, thus allowing the fabrication of smooth adiabatic tapers, by controllably avoiding embedded microspheres formation. Figure 9 shows optical microscope and scanning electron 
microscopy SEM images obtained from SEM instrument JEOL JSM -7401f FESEM- of a tapered microfiber with initial core and cladding diameters of $4 \mu \mathrm{m}$ and $31 \mu \mathrm{m}$, respectively. After the taper fabrication the ends of the fiber must be cleaved to allow its further characterization. The best method for cleaving the fiber ends is by using Focused Ion Beam (FIB) techniques, however in order to avoid this complicated and time consuming method the tapered fibers were cleaved here by using a simple ceramic tile under an optical microscope. It should be stressed that the fact that a simple hand held ceramic tile could be used for cleaving demonstrates the ease of handling as well as the mechanical robustness of these fiber tapers.

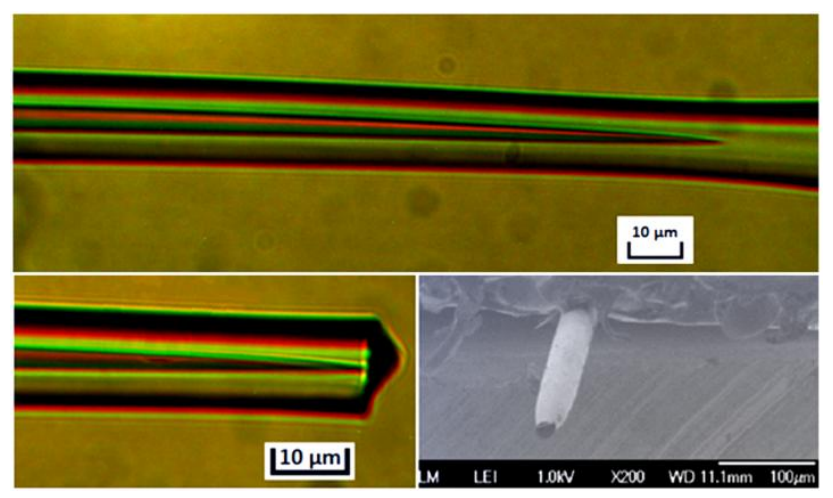

Figure 9. Up: Optical microscope images of a fabricated fiber tip before cleaving. Down: Optical microscope (left) and SEM (right) characteristic image of a fabricated fiber tip after the cleaving process.

The mode profiles of the pristine un-tapered and tapered fibers were extracted using a $60 \mathrm{X}$ objective lens with 0.85 numerical aperture and a scanning slit optical beam profiler (Thorlabs BP209-VIS/M) in order to image the near field intensity distribution at the fiber output. For the excitation a $532 \mathrm{~nm}$ laser is used and light is coupled to the microfiber with a $40 \mathrm{X}$ objective lens with 0.65 numerical aperture. Figure 10 shows a schematic representation of the setup. 


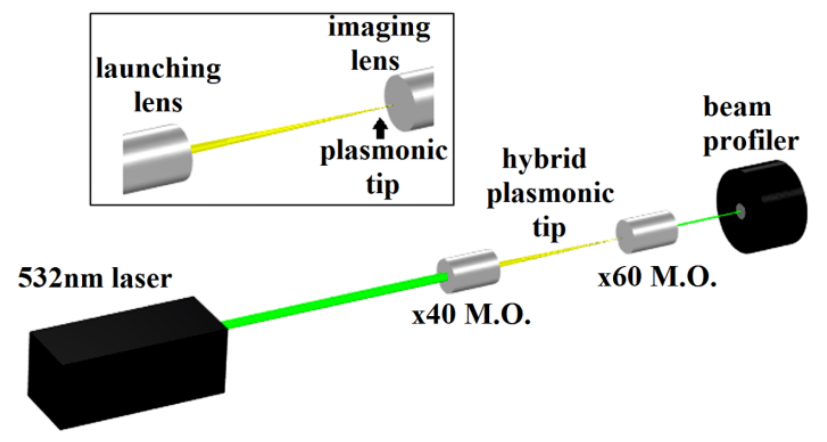

Figure 10. Schematic representation of the characterization setup.

As aforementioned, at the current stage of fiber production the microwires have discontinuous metal cores at long lengths while only limited segments of 3-4 $\mathrm{cm}$ of continuous core were identified for this study. Such discontinuous microwires of relatively long lengths were evaluated also in terms of propagation, and they exhibited uniform and low loss propagation characteristics as demonstrated by coupling with visible light at $671 \mathrm{~nm}$ and $532 \mathrm{~nm}$. Typical gold core microfibers with core and cladding diameters of $4 \mu \mathrm{m}$ and $30 \mu \mathrm{m}$ were butt coupled and visually characterized providing light propagation over a relatively long length of $12 \mathrm{~cm}$ by guiding at microfiber's glass cladding (Figure 11).

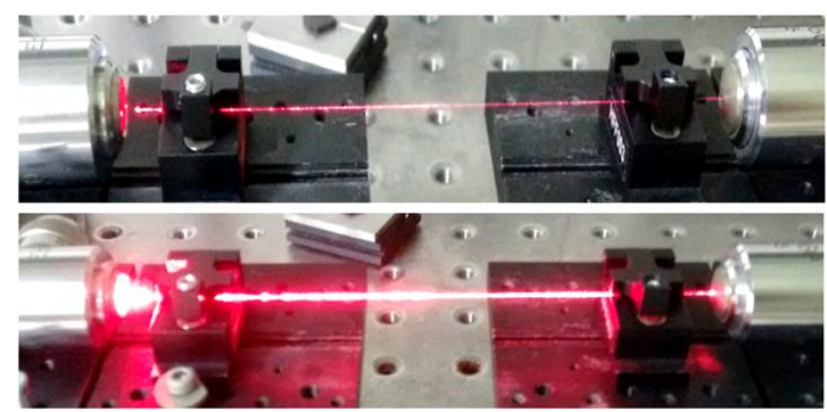

Figure 11. Visible light $(671 \mathrm{~nm})$ propagation in a $12 \mathrm{~cm}$ long segment of discontinuous core microwires, with different input laser power conditions.

In Figure 12 the mode profile of the microfiber before (a) and after (b) the tapering process is presented. The mode profile of the pristine / untapered microfiber (Figure 12 (a)) matches with the profile of the $\mathrm{TM}_{01}$ mode shown in Figure 2 having two intensity peaks corresponding to the gold/glass interface and it was 
obtained after propagation along a $12 \mathrm{~cm}$ long fiber. The conservation and imaging of the plasmon mode at the end face of the microfiber in Figure 11, after such a long propagation length, and despite the plasmon losses and the strong discontinuities of the metal core, is a result of the mode re-excitation mechanism. The relatively high intensity in the middle of the gold core is due to non-optimum cleave performed by a standard ceramic tile. On the contrary the tapered microfiber has one sharp intensity peak (Figure 12 (b) and Figure 13) and matches with the near field calculated from the simulations (Figure 4 (b)).

Near field distribution shown in Fig. $4 \mathrm{~b}$ is theoretically calculated at a position of $20 \mathrm{~nm}$ above the tip apex and has a measured FWHM of $\sim 0.5 \mu \mathrm{m}$. However in the experimental approximate near field measurement a $60 \mathrm{X}$ objective lens was used inducing thus an 60X magnification in the projected near field, resulting in a FWHM of $300 \mu \mathrm{m}$ (as measured from Fig. 12b) and corresponding to an actual FWHM of $300 \mu \mathrm{m} / 60=5 \mu \mathrm{m}$. This disagreement can be easily attributed to lack of experimental control in the micrometer scale of the objective lens positioning relative to tip apex and beam profiler. Such small variations from the optimum focal point position and beam profiler slit can lead to considerable experimental errors. Furthermore in the simulation study only the $\mathrm{TM}_{01}$ is considered while in the real experimental case higher order modes and coupling effects due to possible non-uniformities could be apparent justifying further variations from theory.

Since two separate intensity peaks are not observed in the mode profile of the tapered fiber, the diameter must be less than $380 \mathrm{~nm}$ which is the resolution of the $60 \mathrm{X}$ objective lens that was used for the imaging. The exact diameter of the core at the tip apex though cannot be measured directly by SEM images due to the surrounding dielectric cladding. However, an accurate estimation can be based on the uniform tapering semi-angle of $\sim 3^{\circ}$, by assuming that a $4 \mu \mathrm{m}$ diameter gold core tends to zero after a length of $\sim 38 \mu \mathrm{m}$ resulting in a gold tip with a very small radius surrounded by a large radius silicate glass cladding. 

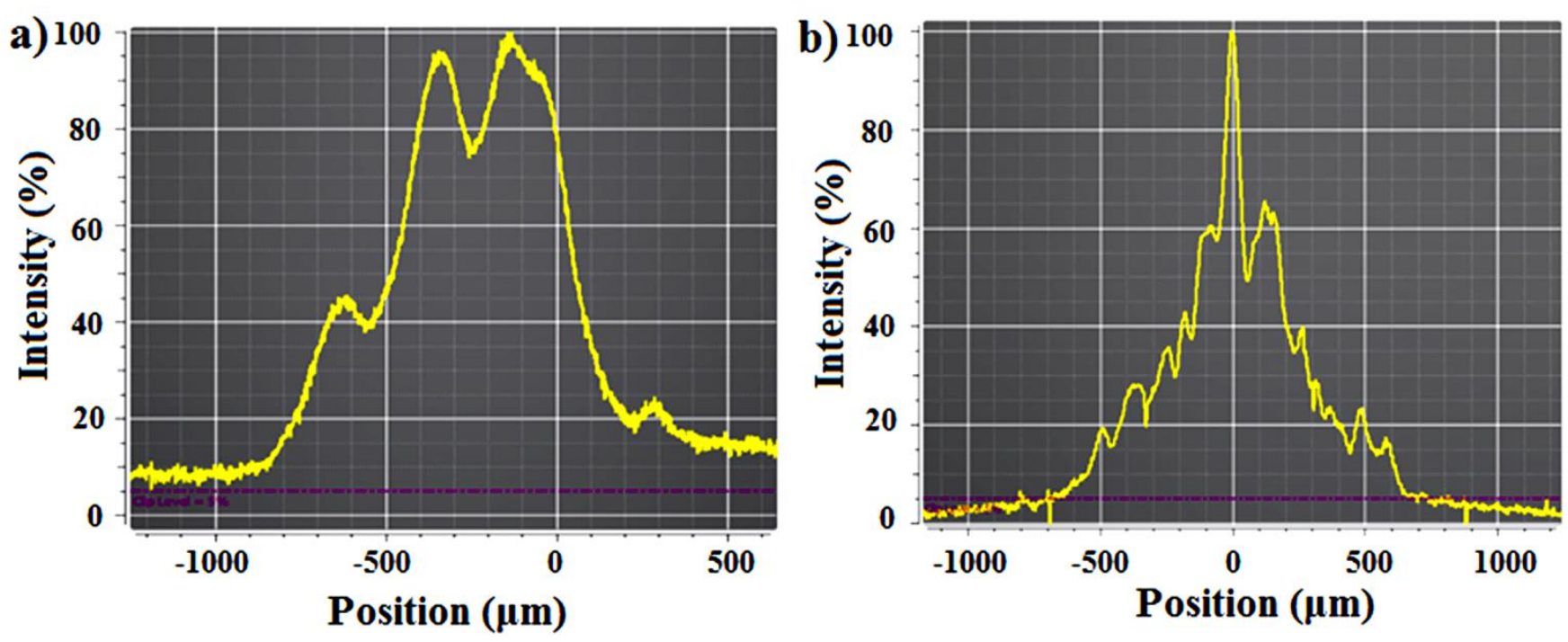

Figure 12. Recorded mode profile of a gold core microfiber before (a) and after $(b)$ the tapering processs.

Please note that in this graph the position 0 corresponds only to the specific beam profiler center, which is irrelevant to the center of microfiber.
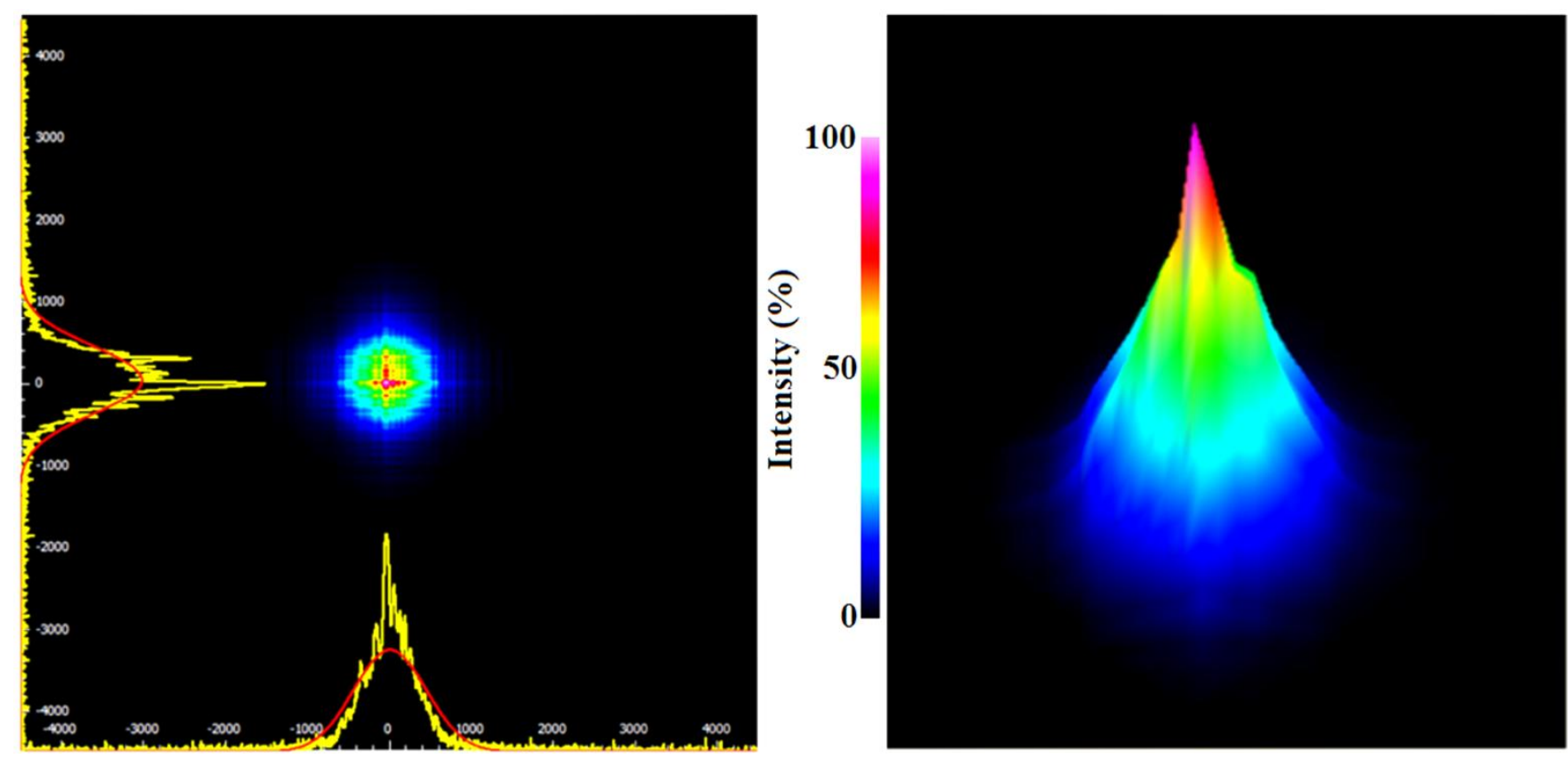

Figure 13. $2 D($ left) and $3 D($ right) recorded field intensity distribution of a tapered gold core microfiber.

The two plots of Figure 12, which correspond to the recorded mode profile of the microfiber before and after the tapering process, cannot be directly compared since with the current characterization 
instrumentation, each beam profiler output is normalized to the maximum intensity. Furthermore and more importantly the coupling conditions can greatly differ for the two fibers before and after tapering, as the method has not yet been standardized.

As discussed above due to the high losses and the short propagation length of $\mathrm{TM}_{01}$ mode, the high intensity at the apex of the tip observed above is due to the continuous re-excitation of the mode from the light coupled in the glass cladding. For metal tips without glass cladding the direct excitation from a fiber would lead to significant losses reducing dramatically the field enhancement and the overall performance of the structure.

\section{CONCLUSIONS}

In this work, a new hybrid platform of metal core/glass cladding microfibers was proposed and investigated both theoretically and experimentally towards the development of robust plasmonic nanotips for light nanofocussing. Borosilicate tubing was chosen for the glass cladding of the microfibers due to the compatibility of its softening point with the melting points of the used metals. The heating and stretching tapering method allowed the successful fabrication of metal tips with the appropriate geometrical characteristics. The ability to control the geometry of the taper ensures the development of adiabatic tips with minimum losses. Furthermore the use of a "fast and cold" tapering approach helps avoid the breaking and collapse of the core during the process by increasing the characteristic break-up time of the metal core and providing thus an adequate time scale for continuous tapering. Theoretical investigations and FEM based microfluidic simulations support the experimental observations and validate the proposed taper fabrication approach. The fabricated tips exhibit high field confinement beyond the diffraction limit at their apex as well as high field enhancement with calculated $E_{f}$ of $10^{5}$. Furthermore, the continuous re-excitation of the fundamental mode due to the repeated total internal reflection of light at the glass/air interface limits the losses induced by the metal leading to high field intensities at the tip apex and thus increases the overall 
performance of the structure and results to even higher $E_{f}$. Additionally, compared to bare metal tips, the presence of the glass cladding offers mechanical robustness as well as ease of light coupling.

\section{ACKNOWLEDGMENTS}

This work was supported in part by the project "Advanced Materials and Devices" (MIS 5002409) which is implemented under the "Action for the Strategic Development on the Research and Technological Sector", funded by the Operational Programme "Competitiveness, Entrepreneurship and Innovation" (NSRF 20142020) and co-financed by Greece and the European Union (European Regional Development Fund). The works was supported in part by the Engineering and Physical Sciences Research Council, (United Kingdom) through the EPSRC Centre for Innovative Manufacturing in Photonics (EP/H02607X/1). COST Action BM1401 "Raman based applications for clinical diagnostics- Raman4Clinics", and COST Action MP1401: "Advanced fibre laser and coherent source as tools for society, manufacturing and lifescience" are also acknowledged. Authors would like also to thank Professor Dimitris Drikakis of Strathclyde Space Institute, University of Strathclyde, UK, for valuable discussions on microfluidic simulations.

\section{REFERENCES}

(1) Stockmann, M. I. Nanoplasmonics: past, present, and glimpse into future. Opt. Express 2011, 19, 22029-22106.

(2) Lee, B.; Lee, I. M.; Kim, S.; Oh, D. H.; Hesselink, L. Review on subwavelength confinement of light with plasmonics. J. Mod. Opt. 2010, 57, 1479-1497.

(3) Di Fabrizio, E.; Schluecker, S.; Wenger, J.; Regmi, R.; Rigneault, H.; Calafiore, G. Roadmap on biosensing and photonics with advanced nano-optical methods. J. Opt. 2016, 18, 063003. 
(4) Sheridan, A. K.; Ngamukot, P.; Bartlett, P. N.; Wilkinson, J. S. Waveguide surface plasmon resonance sensing: Electrochemical desorption of alkane thiol monolayers. Sens. Actuator B-Chem. 2006, 117, 253-260.

(5) Vo-Dinh, T.; Wang, H. N.; Scaffidi, J. Plasmonic nanoprobes for SERS biosensing and bioimaging. J. Biophotonics 2010, 3, 89-102.

(6) Anker, J. N.; Hall, W. P.; Lyandres, O.; Shah, N. C.; Zhao, J.; Van Duyne, R. P. Biosensing with plasmonic nanosensors. Nat. Mater. 2008, 7, 442-453.

(7) Bauch, M.; Toma, K.; Toma, M.; Zhang, Q.; Dostalek, J. Plasmon-enhanced fluorescence biosensors: a review. Plasmonics 2014, 9, 781-799.

(8) Huh, Y. S.; Chung, A. J.; Erickson, D. Surface enhanced Raman spectroscopy and its application to molecular and cellular analysis. Microfluid. Nanofluidics 2009, 6, 285-297.

(9) De Angelis, F.; Das, G.; Candeloro, P.; Patrini, M.; Galli, M.; Bek, A.; Lazzarino, M.; Maksymov, I.; Liberale, C.; Andreani, L. C.; Di Fabrizio, E. Nanoscale chemical mapping using three-dimensional adiabatic compression of surface plasmon polaritons. Nat. Nanotechnol. 2010, 5, 67-72.

(10) Challener, W. A.; Peng, C.; Itagi, A. V.; Karns, D.; Peng, W.; Peng, Y.; Yang, X. M. Heat-assisted magnetic recording by a near-field transducer with efficient optical energy transfer. Nat. Photonics 2009, 3, 220-224.

(11) Wong, C. L.; Olivo, M. Surface plasmon resonance imaging sensors: a review. Plasmonics 2014, 9, 809-824.

(12) Bek, A.; De Angelis, F.; Das, G.; Di Fabrizio, E.; Lazzarino, M. Tip enhanced Raman scattering with adiabatic plasmon focusing tips. Micron 2011, 42, 313-317.

(13) Zervas, M. N. Surface-plasmon polariton waves guided by thin metal-films. Opt. Lett. 1991, 16, 720-722.

(14) Miyata, M.; Takahara, J. Field enhancement by longitudinal compression of plasmonic slow light. J. Appl. Phys. 2012, 111, 053102. 
(15) Albader, S. J.; Imtaar, M. Azimuthally uniform surface plasma modes in thin metallic cylindricalshells. IEEE J. Quantum Electron. 1992, 28, 525-533.

(16) Zhao, Y.; Zhu, L. Coaxial hybrid plasmonic nanowire waveguides. J. Opt. Soc. Am. B 2010, 27 , 1260-1265.

(17) Schmidt, M. A.; Argyros, A.; Sorin, F. Hybrid optical fibers - an innovative platform for in-fiber photonic devices. Adv. Opt. Mater. 2016, 4, 13-36.

(18) Tuniz, A.; Schmidt, M. A. Interfacing optical fibers with plasmonic nanoconcentrators. Nanophotonics 2018, 7, 1279-1298.

(19) Ding, M.; Brambilla, G.; Zervas, M. N. Plasmonic slot nanoresonators embedded in metal-coated plasmonic microfibers. J. Light. Technol. 2013, 31, 3093-3103.

(20) Ding, M.; Zervas, M. N.; Brambilla, G. Transverse excitation of plasmonic slot nano-resonators embedded in metal-coated plasmonic microfiber tips. Appl. Phys. Lett. 2013, 102, 141110.

(21) Petropoulou, A.; Zervas, M. N.; Riziotis, C. Design optimization of gold-coated fiber tips with embedded plasmonic slot nanoresonators. J. Opt. 2017, 19, 055002.

(22) Stockman, M. I. Nanofocusing of optical energy in tapered plasmonic waveguides. Phys. Rev. Lett. 2004, 93, 137404.

(23) Uebel, P.; Bauerschmidt, S. T.; Schmidt, M. A.; Russell, P. S. J. A gold-nanotip optical fiber for plasmon enhanced near-field detection. Appl. Phys. Lett. 2013, 103, 021101.

(24) Zaccaria, R. P.; Alabastri, A.; De Angelis, F.; Das, G.; Liberale, C.; Toma, A.; Giugni, A. Fully analytical description of adiabatic compression in dissipative polaritonic structures. Phys. Rev. B 2012, $86,035410$.

(25) Demming, A. L.; Festy, F.; Richards, D. Plasmon resonances on metal tips: Understanding tipenhanced Raman scattering. J. Chem. Phys. 2005, 122, 184716.

(26) Petropoulou, A.; Antonopoulos, G.; Bastock, P.; Craig, C.; Kakarantzas, G.; Hewak, D. W.; Zervas, M. N.; Riziotis, C. Robust plasmonic tips fabricated by the tapering of composite hybrid silicate 
microfibers with metallic core. In Plasmonics II, Proceedings of SPIE, Beijing, China, Oct 12-14, 2016.

(27) Gholipour, B.; Nalla, V.; Bastock, P.; Khan, K.; Craig, C.; Hewak, D. W.; Zheludev, N. I.; Soci, C. Plasmonic Nanowire Continuum Light Source. In Conference on Lasers and Electro-Optics, Proceedings of CLEO, San Jose, CA, USA, Jun 08-13, 2014.

(28) Kou, F. Y.; Tamir, T. Range extension of surface plasmons by dielectric layers. Opt. Lett. 1987, 12, 367-369.

(29) Leosson, K.; Nikolajsen, T.; Boltasseva, A.; Bozhevolnyi, S. I. Long-range surface plasmon polariton nanowire waveguides for device applications. Opt. Express 2006, 14, 314-319.

(30) Boltasseva, A.; Nikolajsen, T.; Leosson, K.; Kjaer, K.; Larsen, M. S.; Bozhevolnyi, S. I. Integrated optical components utilizing long-range surface plasmon polaritons. J. Light. Technol. 2005, 23, 413422.

(31) Kakarantzas, G.; Leon-Saval, S. G.; Birks, T. A.; Russell, P. S. J. Low-loss deposition of solgelderived silica films on tapered fibers. Opt. Lett. 2004, 29, 694-696.

(32) Birks, T. A.; Li, Y. W. The shape of fiber tapers. J. Light. Technol. 1992, 10, 432-438.

(33) Bastock, P. Manufacturing Novel Fibre. Ph.D. Dissertation, University of Southampton, Southampton, 2015.

(34) Handapangoda, D.; Premaratne, M.; Rukhlenko, I. D.; Jagadish, C. Optimal design of composite nanowires for extended reach of surface plasmon-polaritons. Opt. Express 2011, 19, 16058-16074.

(35) Gramotnev, D. K.; Vogel, M. W.; Stockman, M. I. Optimized nonadiabatic nanofocusing of plasmons by tapered metal rods. J. Appl. Phys. 2008, 104, 034311.

(36) Wiener, A.; Fernandez-Dominguez, A. I.; Horsfield, A. P.; Pendry, J. B.; Maier, S. A. Nonlocal effects in the nanofocusing performance of plasmonic tips. Nano Lett. 2012, 12, 3308-3314.

(37) Jiang, R. H.; Chen, C.; Lin, D. Z.; Chou, H. C.; Chu, J. Y.; Yen, T. J. Near-field plasmonic probe with super resolution and high throughput and signal-to-noise ratio. Nano Lett. 2018, 8, 881-885. 
(38) Zhang, W.; Cui, X.; Martin, O. J. F. Local field enhancement of an infinite conical metal tip illuminated by a focused beam. J. Raman Spectrosc. 2009, 40, 1338-1342.

(39) Shabahang, S.; Kaufman, J. J.; Deng, D. S.; Abouraddy, A. F. Observation of the Plateau-Rayleigh capillary instability in multi-material. Appl. Phys. Lett. 2011, 99, 161909.

(40) Aktas, O.; Ozgur, E.; Tobail, O.; Kanik, M.; Huseyinoglu, E.; Bayindir, M. A new route for fabricating on-chip chalcogenide microcavity resonator arrays. Adv. Opt. Mater. 2014, 2, 618-625.

(41) Mead-Hunter, R.; King, A. J. C.; Mullins, B. J. Plateau Rayleigh instability simulation. Langmuir 2012, 28, 6731-6735.

(42) Alchalaby, A.; Lwin, R.; Al-Janabi, A. H.; Trimby, P. W.; Fleming, S. C.; Kuhlmey, B. T.; Argyros, A. Investigation of Plateau-Rayleigh instability in drawn metal-polymer composite fibres for metamaterials fabrication. J. Light. Technol. 2016, 34, 2198-2205.

(43) Kaufman, J. J.; Ottman, R.; Tao, G.; Shabahang, S.; Banaei, E. H.; Liang, X.; Johnson, S. G.; Fink, Y.; Chakrabarti, R.; Abouraddy, A. F. In-fiber production of polymeric particles for biosensing and encapsulation. Proc. Natl. Acad. Sci. U.S.A. 2013, 110, 15549-15554.

(44) Tomotika, S. On the instability of a cylindrical thread of a viscous liquid surrounded by another viscous fluid. Proc. Royal Soc. Lond. A 1935, 150, 322-337. 


\section{TOC graphic}

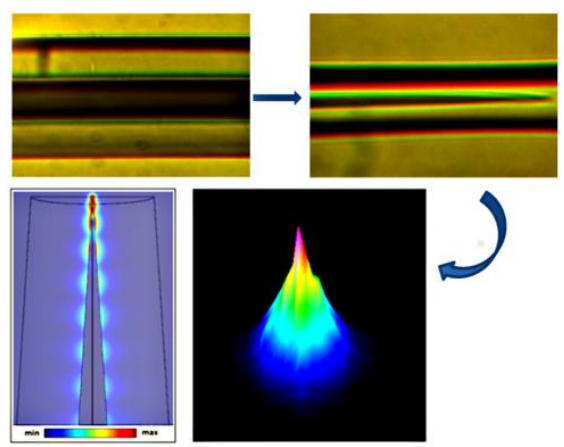

